\title{
La Salud Familiar al servicio de la Odontología
}

\section{Sr. Editor:}

La familia constituye la primera red de apoyo social que posee el individuo y se reconoce su función protectora ante las tensiones que genera la vida ${ }^{1}$; es también el principal recurso de promoción en salud y prevención de la enfermedad y cumple una función cuidadora y protectora de sus miembros, sobre todos aquellos que son enfermos y dependientes.

Con frecuencia, cuando los pacientes consultan, ya los procesos se encuentran avanzados y algunas veces se requiere de los miembros de la familia para la solución y pronostico de la enfermedad, por ser el contexto en donde permanece la información referente a la evolución de la enfermedad y la adherencia al tratamiento ${ }^{2}$. Por ello se busca emplear el recurso familiar como estrategia de cambio hacia el manejo integral de la enfermedad y no sólo orientar el ejercicio profesional hacia el individuo. De allí nace la Salud Familiar (SF), una transdisciplina que ofrece una atención integral en salud, con un nuevo enfoque -complementario del individual y del comunitario- en la formación del personal de salud ${ }^{3}$. En algunos profesionales de la salud, como los odontólogos, se genera cierta resistencia a brindar la atención bajo este enfoque, dado que la formación que reciben es un tanto individualista, pero está claro que cuanto mayor sea la formación del profesional hacia el área social, más se incrementa la aceptación de este modelo de atención.

En Colombia, específicamente en Cartagena, la Universidad de Cartagena, dentro del programa de Odontología, utiliza la SF como transdisciplina, buscando generar bases cognoscitivas y humanísticas para identificar y hallar soluciones acertadas a problemas asociados con la salud del individuo en su entorno familiar y social. Al interactuar con la comunidad, se logra una mejor identificación y análisis de los factores de riesgo relacionados con el proceso salud-enfermedad, buscando su control o eliminación al promover el autocuidado en salud. Se revisan temas relacionados con nociones generales de la SF, ciclo vital individual y familiar, estructura familiar, atención odontológica con enfoque de riesgo, historias clínicas de SF (instrumentos de SF: familiograma, ecomapa, Apgar familiar) que evalúa los recursos como el apoyo social y la funcionalidad familiar; actualiza conceptos acordes a las demandas del medio como maltrato infantil y violencia intrafamiliar, familias con discapacitados, fomento de la lactancia materna (estrategia IAMI) ${ }^{4}$ y educación en enfermedades prevalentes en la infancia (estrategia AIEPI) ${ }^{5}$ y relaciona la salud bucal a dinámicas como pobreza y miseria y calidad de vida 6 . Por otro lado trabaja en el programa "Odontología con enfoque en Salud Familiar" implementado en comunidades vulnerables, escenarios de prácticas, donde se evalúan las competencias adquiridas por los estudiantes.

A nivel de las clínicas odontológicas, todo paciente se valora por los componentes de semiología, periodoncia, rehabilitación, forense y se innova a través de la atención bajo el enfoque de riesgo desde la $\mathrm{SF}^{7}$, diligenciando la historia clínica de SF para evaluar factores de riesgo y protectores del paciente y su familia, con el fin de elaborar guías preventivas y desarrollar intervenciones en promoción de la salud y prevención de la enfermedad, mediante estrategias de consejería, filtrado y medidas específicas de protección ${ }^{8}$. Esto ha logrado que, desde la práctica odontológica, se actúe sobre diferentes factores de riesgo y se anticipe a la presencia de ciertas enfermedades como hipertensión, diabetes y estrés, al realizar educación a los pacientes en temas como obesidad, sedentarismo, estrés, dependencia emocional y física. Incluso se orienta en situaciones familiares como el divorcio, demandas por alimentos, o afiliaciones al sistema de seguridad social. Se busca que se modifiquen estilos de vida en relación a la salud física y mental, fomentando desde la odontología la realización de ejercicio, el aumento de la ingesta de agua o una dieta equilibrada acorde al estado sistémico del paciente. Se persigue también mejorar la autoestima, buscando apoyo en las demás disciplinas de la salud y generando una atención odontológica transdisciplinaria "más allá de la boca".

Los cambios de comportamientos de riesgo por adquisición de factores protectores por el paciente, se observan mediante seguimiento semanal y bimestral.

Este modelo de atención ("Odontología con enfoque en Salud Familiar"), logra mejorar la calidad de vida de los pacientes en el plano biosicosocial; estos terminan su tratamiento 
odontológico con una mejor autoestima, con factores de riesgos controlados o eliminados y con un aumento en factores protectores para su salud. El modelo necesita seguimiento y evaluación para evaluar su impacto y su posible implementación en otras instituciones de salud, contribuyendo de esta forma no solo a mejorar los índices de morbilidad bucal sino también a disminuir la morbimortalidad de los pacientes adultos y fomentando personas sanas y productivas para el país, desde la atención odontológica.

\section{BIBLIOGRAFÍA}

1. Novilla M, Lelinneth B, Barnes M. De La Cruz N, Williams P, Rogers J. Public Health Perspectives on the Family: An Ecological Approach to Promoting Health in the Family and Community. Fam Community Health. .2006; 29(1): 28-42.

2. Florenzano R, Horwitz N. Family health: conceptual development and its application. Project of primary health care and general family medicine. Cuad Med.-Soc. (Santiago de Chile).1983;24(4):149-155.

3. García-Huidobro D. Enfoque familiar en la Atención Primaria: Una propuesta para mejorar la salud de todos. Rev Méd Chile. 2010; 138(11):1463-1464.

4. Iniciativa Instituciones Amigas de la Mujer y de la Infancia. Disponible en: http://www.publicaciones.unicefcolombia. com/wp-content/uploads/2011/03//AMl-1.pdf

5. Manual Clínico para el Aprendizaje de AIEPI en Enfermería (Atención Integrada de las Enfermedades Prevalentes de la Infancia. Disponible en: http://new.paho.org/per/images/ stories/PER/Documentos/manual\%20cl\%EDnico.pdf
6. Tuesca R. La Calidad de vida, su importancia y como medirla. Salud Uninorte. Barranquilla (Col.) 2005; 21: 7686. Disponible en: http://ciruelo.uninorte.edu.co/pdf/ salud_uninorte/21/8_La\%20Calidad\%20de\%20Vida.pdf

7. Louro Bernal I. Modelo de Salud del grupo Familiar. Rev cubana de Salud Publica. 2005; 31(4). Disponible en: http://bvs.sld.cu/revistas/spu/vol31_4_05/spu11405.htm

8. Pineda C. Guías preventivas por ciclo vital individual en Colombia. Colombia Médica 2006; 37 (2). Disponible en: URL: http://redalyc.uaemex.mx/pdf/283/28337911.pdf

\section{Shyrley Díaz Cárdenas ${ }^{1}$, Lesbia Rosa Tirado Amador $^{2}$, Meisser Vidal Madera Anaya ${ }^{3}$}

Odontóloga, Magister en Salud Pública, Especialista en Salud Familiar y Gerencia en Salud Docente Facultad de Odontología.

${ }^{2}$ Odontóloga, Joven Investigadora de Colciencias y Rural de Investigaciones.

${ }^{3}$ Odontólogo, Candidato a Maestría de Bioquímica, Joven Investigador de Colciencias

Dpto. Odontologia Preventiva y Social, Dpto. Investigaciones. Facultad de Odontología, Universidad de Cartagena de Indias. Correo electrónico: shyrley77@hotmail.com 